\title{
Artículos
}

\section{Los salvadoreños evalúan los tres años de gobierno de Calderón}

\author{
Instituto Universitario de Opinión Públical
}

\section{Resumen}

A finales del mes de mayo de 1997, el IUDOP llevó a cabo una encuesta de opinión pública con una muestra de 1,217 adultos salvadoreños, representativa de la población de todo el país y con un margen de error de más-menos cuatro por ciento. El sondeo, realizado dentro de la serie de evaluaciones anuales sobre el gobierno, que realiza la Universidad Centroamericana "José Simeón Cañas", mostró que la mayor parte de los ciudadanos mantiene una visión negativa de la administración de Calderón Sol, a la cual califican con una nota promedio de 4.97 y le atribuyen la falta de solución de los problemas económicos y de seguridad pública.

\section{Introducción}

El tercer año de la presidencia de Armando Calderón Sol ha concluido. Como es costumbre, el IUDOP ha llevado a cabo una encuesta de opinión pública para conocer los juicios de la ciudadanía salvadoreña acerca del segundo gobierno de ARENA, en cuanto a las evaluaciones globales sobre temas específicos como la economía, la delincuen-

1 En la redacción de este artículo colaboró el señor Peter Moore, del Departamento de Ciencias Políticas de la Universidad de lowa, a quien el IUDOP quiere agradecer profundamente. 
cia y la corrupción. Los datos que se exponen en este artículo pueden parecer repetitivos en comparación con los obtenidos en evaluaciones anteriores, sobre todo en la de 1996, y en cierta forma lo son. Sin embargo, esto indica que, de manera global, la percepción social y la opinión pública sobre el desempeño del gobierno no han variado de manera sustancial. Los salvadoreños ya tienen un juicio relativamente estable sobre su propio gobierno, de alguna manera la opinión pública se ha sedimentado alrededor de una visión específica que acusa a la administración Calderón Sol de los problemas económicos y de seguridad pública (más de los primeros que de los segundos). No obstante, lo que sí parece característico de esta evaluación es la intensidad con la cual los salvadoreños critican a la política económica del gobierno. Ahora, más que en los años anteriores, la población cree que el gobierno no sólo hace muy poco para resolver los problemas económicos del país, sino que, además, es el principal responsable de los mismos.

La información brindada por la encuesta de mayo del IUDOP no sólo es útil para entender la visión de los ciudadanos hacia su propio gobierno, sino también para encontrar explicaciones a la conducta electoral de la población en los comicios de este año. El sondeo muestra de fondo la insatisfacción de amplios sectores de la población, sobre todo, los más desposeídos, con la forma en que se conduce el país, parte de ese desencanto fue el que precisamente se expresó en las urnas y fuera de ellas.

\section{Encuesta y población encuestada}

Al concluir el tercer año del gobierno de presidente Armando Calderón Sol, el Instituto de Opinión Pública (IUDOP) de la Universidad Centroamericana "José Simeón Cañas" realizó un sondeo para conocer la opinión de la población salvadore- ña sobre los tres años de gestión y para ofrecerles a los ciudadanos la oportunidad de expresar su opinión acerca del trabajo que desempeñan los poderes ejecutivo y legislativo de El Salvador. La encuesta contempla, además, los factores que estuvieron detrás de la conducta electoral en los pasados comicios, sin embargo, por razones temáticas, esta parte no será abordada en el presente artículo.

La encuesta se realizó entre el 17 y el 25 de mayo del presente año, en los catorce departamentos de la República, con representatividad nacional en cuanto a todas las características demográficas del país. En la parte de la evaluación del gobiemo se usó una boleta autocodificable, por lo cual no fue necesario el trabajo adicional de codificar las respuestas porque las opciones de respuesta y sus valores se marcaban en el momento en que los encuestadores del IUDOP realizaban la entrevista. La mayoría de las preguntas de la encuesta fue cerrada, excepto una pregunta sobre la principal tarea del gobierno en su próximo año de gestión, la cual se dejó totalmente abierta.

El cuestionario usado para el sondeo de evaluación del tercer año de gestión del Ejecutivo fue preparado sobre la misma base de preguntas usada con anterioridad para realizar las evaluaciones periódicas gubernamentales. El sondeo sobre la evaluación del gobierno consistía básicamente en tres componentes. La primera parte recogía los datos relacionados con la muestra y correspondía a la información sobre el departamento y municipio de residencia y el estrato socioeconómico del encuestado. En segundo lugar, se recogía, de los entrevistados, información general de tipo demográfico, incluyendo género, edad, nivel de estudios, situación de trabajo, tipo de trabajo y religión. Finalmente, la tercera parte del cuestionario consistía en veintidós preguntas sobre el trabajo del gobierno nacional, además de otros temas relaconados con el mismo. 
Concretamente, los temas abordados en la encuesta son: el principal problema del país, evaluación del desempeño del gobierno, cambios positivos y negativos producto de la gestión gubernamental, cumplimiento de promesas, combate a los problemas de corrupción, delincuencia y economía, grupo más beneficiado por las políticas del gobierno, situación familiar y del país con la actual administración, proceso de privatización de empresas públicas, evaluación del trabajo de la asamblea legislativa, tarea principal que debe enfrentar el gobierno en el futuro, rumbo general del país $y$, para terminar, las declaraciones de voto para alcalde municipal y diputado departamental en las recientes elecciones.

Como ya se ha dicho, la muestra diseñada para este sondeo tiene una representación nacional. Se realizó en todos los departamentos de la república salvadoreña: Ahuachapán, Santa Ana, Sonsonate, La Libertad, Chalatenango, San Salvador, Cabañas, Cuscatlán, San Vicente, La Paz, Usulután, San Miguel, Morazán y La Unión. En cada departamento, la muestra se dividió para representar las características demográficas de la población misma. El sondeo se dividió primero en dos grandes sectores: urbano y rural. El primero de estos sectores fue dividido en cinco estratos socioeconómicos, es decir, alto, medio-alto, medio-bajo, obrero y marginal. El sector rural se dejó como categoría única por la dificultad en encontrar criterios de tipificación. En el muestreo se consideraron también cuotas representativas de la población por sexo y edad, de tal manera que cada boleta estaba marcada con las características que debía cumplir el encuestado para ser tomado en cuenta dentro de la investigación.

\section{Cuadro 1}

Distribución de la población encuestada según departamento y sector social

\begin{tabular}{|c|c|c|c|c|c|c|c|c|}
\hline \multirow[b]{2}{*}{ Departamento } & \multirow[b]{2}{*}{ Alto } & \multirow[b]{2}{*}{ Medio-alto } & \multicolumn{2}{|c|}{ Sector social } & \multirow[b]{2}{*}{ Marginal } & \multirow[b]{2}{*}{ Rural } & \multicolumn{2}{|c|}{ Todos } \\
\hline & & & Medio-bajo & Obrero & & & $\mathbf{N}$ & $\%$ \\
\hline Ahuachapán & 3 & 4 & 4 & 7 & 4 & 42 & 64 & 5.3 \\
\hline Santa Ana & 7 & 13 & 13 & 21 & 13 & 42 & 109 & 9.0 \\
\hline Sonsonate & 4 & 10 & 10 & 15 & 10 & 35 & 84 & 6.9 \\
\hline La Libertad & 11 & 15 & 15 & 23 & 17 & 48 & 129 & 10.6 \\
\hline Chalatenango & 4 & 4 & 3 & 8 & 6 & 22 & 47 & 3.9 \\
\hline San Salvador & 28 & 64 & 61 & 91 & 59 & 47 & 350 & 28.8 \\
\hline Cabañas & 2 & 3 & 3 & 5 & 3 & 16 & 32 & 2.6 \\
\hline Cuscatlán & 2 & 5 & 5 & 8 & 5 & 15 & 40 & 3.3 \\
\hline San Vicente & 2 & 4 & 4 & 6 & 4 & 12 & 32 & 2.6 \\
\hline La Paz & 3 & 9 & 8 & 12 & 8 & 19 & 59 & 4.8 \\
\hline Usulután & 4 & 8 & 8 & 13 & 4 & 38 & 75 & 6.2 \\
\hline San Miguel & 6 & 15 & 14 & 21 & 14 & 21 & 91 & 7.5 \\
\hline Morazán & 1 & 5 & 6 & 9 & 5 & 20 & 46 & 3.8 \\
\hline La Unión & 4 & 6 & 6 & 10 & 5 & 28 & 59 & 4.8 \\
\hline \multirow[t]{3}{*}{ Todos } & 81 & 165 & 160 & 249 & 157 & 405 & 1217 & - \\
\hline & & & & & & & & \\
\hline & 6.7 & 13.6 & 13.1 & 20.5 & 12.9 & 33.3 & - & 100.0 \\
\hline
\end{tabular}


La aplicación del cuestionario se hizo por aproximación no sistemática a los hogares ubicados en los municipios y las zonas definidas de manera aleatoria. Los encuestadores explicaban a las personas abordadas los objetivos y el tema de la encuesta. Se entrevistó únicamente a personas que quisieran contestar y cumplieran con los requerimientos descritos en la boleta para completar la cuata muestral (estrato, sexo y edad), y sólo a una persona por hogar. La muestra final obtenida fue de 1,217 encuestas válidas, con un margen de error estimado de +/- 0.04 (4 por ciento). El 48.2 por ciento de los encuestados pertenece al sexo masculino, mientras que el 51.8 por ciento corresponde al sexo femenino. La edad mínima de los encuestados fue de 18 años, y la edad máxima, de 80 años; la edad promedio de los entrevistados fue de 37.5 años con una desviación típica de 14.7 años. El Cuadro 1 muestra la distribución de la población encuestada por departamentos y sector social.

\section{La evaluación del trabajo del Ejecutivo}

La mayor parte del sondeo se refirió a la evaluación que los salvadoreños realizaron sobre el desempeño del Ejecutivo en sus tres años de gestión. Para sacar una evaluación completa del actual gobierno de ARENA, la encuesta se dividio en varias partes que abordaban diversos temas de interés en cuanto a la política del gobierno. A continuación se presentan los resultados según dicho esquema. En primer lugar, se detallan los puntos tanto positivos como negativos de la gestión gubernamental; en segundo lugar, se presenta la opinión de los ciudadanos sobre el trabajo del gobierno en las tres áreas problemáticas del país en la actualidad: delincuencia, economía y corrupción; se sigue con las opiniones sobre el cumplimiento de las promesas. Luego, se muestran las evaluaciones generales, tanto del desempeño del gobierno en su conjunto como el trabajo de la cabeza del mismo, esto es, el Presidente. El siguiente tema tiene que ver con el rumbo general del país en la actualidad. Finalmente, se discuten los resultados de esta encuesta en comparación con las evaluaciones gubernamentales anteriores del IUDOP (cien días, un año y dos años).

\subsection{Lo positivo del gobierno}

¿Han habido cambios positivos durante la gestión del presidente Armando Calderón Sol? De acuerdo con el Cuadro 2, poco más de la mitad de los encuestados, es decir, el 55.4 por ciento, dice que no han habido o, por lo menos, no los logran identificar. Por el contrario, el 42.1 por ciento de los entrevistados sí ha notado cambios positivos con la actual presidencia del gobierno. En el Cuadro 2 se observa cómo las percepciones de cambios positivos cambian entre los distintos grupos sociales y educativos. En cuanto a los estratos socioeconómicos, los más dispuestos a considerar que el Presidente Calderón Sol ha logrado cambios positivos son los de la clase alta (49.4 por ciento), casi la mitad. De manera semejante, las personas que tienen un nivel de estudios superior (técnico o licenciatura) están más dispuestas responder de manera positiva (47.8 por ciento). En cambio, los ciudadanos que ven menos cambios positivos pertenecen al estrato obrero ( 36.1 por ciento) y a quienes no poseen formación educativa alguna (35.2 por ciento).

En cuanto a los cambios positivos específicos (Cuadro 3) notados por los ciudadanos salvadoreños durante el gobierno actual, la respuesta más sobresaliente fue el arreglo de las calles (24.9 por ciento de las respuestas). Este asunto, que puede resultar curioso, no tiene que ver tanto con el bienestar de las mayorías, ni con las reformas económicas ni políticas ni de seguridad que necesita nuestra sociedad, sino que está relacionado con la impresión tangible de una cuarta parte de ciudadanos sobre los cambios ornamentales operados en las vías y los centros públicos. Es decir, a tres 
años de gestión, los salvadoreños no se refieren a $\mid$ como los principales logros del Ejecutivo, sino las políticas económicas o de seguridad pública que se refieren a los cambios en el ornato público.

Cuadro 2

¿Ha notado usted algún cambio positivo en nuestro país desde que entró el actual gobierno de ARENA?

(En porcentajes)

\begin{tabular}{llcc}
\hline \multicolumn{2}{c}{ Ha notado cambios positivos } & & \\
Variables & No & Si & No sabe \\
\hline Estrato & & & \\
Alto & 48.1 & 49.4 & 2.5 \\
Medio-alto & 54.5 & 42.4 & 3.0 \\
Medio-bajo & 58.1 & 38.8 & 3.1 \\
Obrero & 61.4 & 36.1 & 2.4 \\
Marginal & 54.8 & 43.3 & 1.9 \\
Rural & 52.6 & 44.9 & 2.5 \\
Estudios & & & \\
Ninguno & 60.5 & 35.2 & 2.3 \\
Primaria & 56.0 & 41.6 & 2.4 \\
Plan básico & 55.8 & 41.9 & 2.3 \\
Bachillerato & 56.6 & 41.3 & 1.9 \\
Superior & 50.4 & 47.8 & 2.5 \\
Todos & 55.4 & 42.1 & \\
\hline
\end{tabular}

\section{Cuadro 3}

Opinión sobre el principal logro del gobierno en los tres años de gestión según variables

(En porcentajes)

\begin{tabular}{|c|c|c|c|c|c|c|c|c|c|c|}
\hline \multirow[b]{2}{*}{ Variables } & \multirow[b]{2}{*}{ Ninguno } & \multirow[b]{2}{*}{$\begin{array}{l}\text { Arreglo } \\
\text { de calles }\end{array}$} & \multicolumn{5}{|c|}{ Principal logro } & \multirow[b]{2}{*}{ pobres } & \multirow[b]{2}{*}{ Otras } & \multirow[b]{2}{*}{ No sabe } \\
\hline & & & $\begin{array}{l}\text { Cumplir } \\
\text { acuerdos }\end{array}$ & $\begin{array}{l}\text { Servicios } \\
\text { púbicos }\end{array}$ & $\begin{array}{l}\text { Medidas } \\
\text { económicos }\end{array}$ & $\begin{array}{l}\text { Combatir } \\
\text { delincuencia }\end{array}$ & $\begin{array}{l}\text { Ayudar a } \\
\text { Educación }\end{array}$ & & & \\
\hline \multicolumn{11}{|l|}{ Estrato } \\
\hline Alto & 22.2 & 22.2 & 2.5 & 11.1 & 7.4 & 2.5 & 2.5 & 3.7 & 11.1 & 14.8 \\
\hline Medio-alto & 31.5 & 17.6 & 11.5 & 6.7 & 6.1 & 1.8 & 3.0 & 1.8 & 12.1 & 7.9 \\
\hline Medio-bajo & 35.0 & 22.5 & 5.0 & 5.0 & 4.4 & 2.5 & 4.4 & 1.3 & 11.3 & 8.8 \\
\hline Obrero & 31.7 & 22.9 & 5.2 & 4.4 & 3.2 & 2.4 & 0.8 & 2.8 & 7.6 & 18.9 \\
\hline Marginal & 26.1 & 35.7 & 4.5 & 7.0 & 3.8 & 4.5 & 1.9 & 0.6 & 3.2 & 12.7 \\
\hline Rural & 21.0 & 26.4 & 8.9 & 8.4 & 3.7 & 4.9 & 2.7 & 2.7 & 6.7 & 14.6 \\
\hline \multicolumn{11}{|l|}{ Estudios } \\
\hline Ninguno & 24.1 & 23.5 & 6.2 & 9.3 & 3.7 & 4.9 & 1.9 & 3.1 & 4.3 & 19.1 \\
\hline Primaria & 25.5 & 26.6 & 7.3 & 6.5 & 4.3 & 4.1 & 1.9 & 1.1 & 6.3 & 16.3 \\
\hline Plan básico & 31.4 & 25.6 & 5.8 & 7.0 & 1.2 & 2.9 & 3.5 & 2.3 & 7.6 & 12.8 \\
\hline Bachillerato & 31.8 & 28.9 & 5.4 & 7.4 & 1.2 & 2.9 & 2.1 & 3.3 & 6.6 & 10.3 \\
\hline Superior & 24.6 & 19.4 & 9.3 & 5.6 & 9.3 & 2.6 & 3.4 & 1.9 & 14.6 & 9.3 \\
\hline Todos & 27.2 & 24.9 & 7.0 & 6.9 & 4.3 & 3.5 & 2.5 & 2.2 & 8.1 & 13.6 \\
\hline
\end{tabular}

¿Podría mencionar el principal logro del gobierno en estos tres años? 
Ahora bien, considerando los cambios positivos menos superficiales y de más importancia para la mayoría de los ciudadanos, hubo otros temas que salieron de la encuesta del IUDOP, como los logros del gobierno actual de Alianza Republicana Nacionalista. El 7 por ciento de la población considera la conclusión del proceso de cumplimiento de los Acuerdos de Paz como un logro positivo de esta gestión gubernamental, mientras que un porcentaje parecido, el 6.9 por ciento, señala el mejoramiento de los servicios públicos (como agua, luz, teléfono) como una fuente de cambio positivo. Muy pocos consideraron que el gobierno actual ha conseguido frutos positivos en cuanto a las reformas económicas (4.3 por ciento), el combate de la delincuencia ( 3.5 por ciento) u otros temas que resultan importantes para los salvadoreños (ver la discusión de la tarea principal que debe enfrentar el gobierno y el Cuadro 10 que siguen a continuación).

\subsection{Lo negativo del gobierno}

El sondeo del IUDOP también abordó los cambios negativos con la gestión del presidente Ar- mando Calderón Sol. Los resultados se presentan en el Cuadro 4. La mayoría de los encuestados, o sea, el 65.2 por ciento, notó cambios negativos desde que entró el actual gobierno de ARENA, mientras que sólo el 27.9 por ciento opinó lo contrario. Así como con los logros, es interesante identificar cuáles grupos están más dispuestos a considerar que sí han habido cambios negativos en el país en estos tres años. En cuanto a esta situación, es muy llamativo que los estratos medios y quienes poseen mayor educación formal consideraran con más frecuencia que han habido cambios negativos. De hecho, los estratos medio-alto y medio-bajo contestaron con más frecuencia que sí han ocurrido transformaciones negativas o fracasos en el país (el 78.2 por ciento y el 75.6 por ciento, respectivamente). En el área rural, por su parte, el 57.8 por ciento notó cambios negativos por parte del gobierno actual. Un dato sobresaliente, que resultó de comparar las respuestas de las personas con distintos niveles educativos, fue que el 77.2 por ciento de los encuestados, con un nivel superior de educación, notó fracasos en comparación con el 57.4 por ciento de los entrevistados que carecían de educación formal.

\section{Cuadro 4}

¿Ha notado usted algún cambio negativo en nuestro país desde que entró el actual gobierno de ARENA?

(En porcentajes)

\begin{tabular}{llll}
\hline & \multicolumn{2}{c}{ Ha notado cambios negativos } & \\
\cline { 2 - 4 } Variables & No & Sí & No sabe \\
\hline Estrato & & & \\
Alto & 30.9 & 65.4 & 3.7 \\
Medio-alto & 17.0 & 78.2 & 4.8 \\
Medio-bajo & 21.3 & 75.6 & 3.1 \\
Obrero & 31.3 & 62.7 & 6.0 \\
Marginal & 28.7 & 64.3 & 7.0 \\
Rural & 31.9 & 57.8 & 10.4 \\
& & & \\
Estudios & & 57.4 & 8.6 \\
Ninguno & 34.0 & 60.3 & 9.0 \\
Primaria & 30.7 & 57.4 & 7.6 \\
Plan básico & 37.8 & 71.9 & 5.8 \\
Bachillerato & 22.3 & 77.2 & 3.7 \\
Superior & 19.0 & 65.2 & 6.9 \\
Todos & & & \\
\hline
\end{tabular}


El Cuadro 5 detalla las transformaciones negativas específicas que la gente ha notado desde que entró el actual gobierno de ARENA. El aspecto más señalado como un fracaso por parte del gobierno es el elevado nivel de inflación que sufre actualmente El Salvador, esto fue destacado por el 28.1 por ciento. También es de importantcia para los salvadoreños el problema del crimen, que provocó que el 15.2 por ciento respondiera que el aumento de la delincuencia ha sido el fracaso princi- pal de la gestión de Calderón Sol. Del resto de respuestas sobre los cambios negativos en el país, la mayoría aborda asuntos económicos como el desempleo (11.6 por ciento), la preocupación del gobierno sólo por los ricos (6.9 por ciento), el aumento del Impuesto al Valor Agregado (5.5 por ciento) y la privatización (4.3 por ciento). Finalmente, el 3.1 por ciento mencionó "más corrupción" como el principal fracaso de estos tres años de gestión del Presidente Calderón Sol.

\section{Cuadro 5}

Opinión sobre el principal fracaso del gobierno en los tres años de gestión según variables (En porcentajes)

\begin{tabular}{|c|c|c|c|c|c|c|c|c|c|c|}
\hline \multirow[b]{2}{*}{ Variables } & \multicolumn{10}{|c|}{ Principal fracaso } \\
\hline & Ninguno & Inflación & $\begin{array}{c}\text { Aumento } \\
\text { delincuencia }\end{array}$ & Desempleo & $\begin{array}{l}\text { Preocupación } \\
\text { por los ricos }\end{array}$ & $\begin{array}{l}\text { Aumento } \\
\text { IVA }\end{array}$ & Privatización & $\begin{array}{c}\text { Más } \\
\text { comupción }\end{array}$ & Otras & No sabe \\
\hline \multicolumn{11}{|l|}{ Estrato } \\
\hline Alto & 6.2 & 34.6 & 17.3 & 3.7 & 9.9 & 2.5 & 2.5 & 0.0 & 11.1 & 12.3 \\
\hline Medio-alto & 4.2 & 20.0 & 17.6 & 12.1 & 10.3 & 7.3 & 5.5 & 7.3 & 9.7 & 6.1 \\
\hline Medio-bajo & 3.1 & 30.6 & 18.1 & 8.1 & 6.9 & 5.6 & 4.4 & 5.0 & 10.0 & 8.1 \\
\hline Obrero & 2.8 & 26.5 & 15.7 & 13.7 & 3.6 & 5.2 & 6.8 & 3.2 & 7.6 & 14.9 \\
\hline Marginal & 0.6 & 28.7 & 12.1 & 12.1 & 10.8 & 4.5 & 7.0 & 1.3 & 8.9 & 14.0 \\
\hline Rural & 5.7 & 29.9 & 13.6 & 12.8 & 5.4 & 5.9 & 1.5 & 2.0 & 6.2 & 17.0 \\
\hline \multicolumn{11}{|l|}{ Estudios } \\
\hline Ninguno & 6.8 & 31.5 & 20.4 & 14.2 & 3.1 & 1.9 & 1.9 & 0.6 & 3.1 & 16.7 \\
\hline Primaria & 4.1 & 31.3 & 11.7 & 10.6 & 6.0 & 6.5 & 3.3 & 2.4 & 6.3 & 17.9 \\
\hline Plan básico & 3.5 & 19.8 & 15.1 & 16.3 & 8.7 & 6.4 & 4.1 & 1.2 & 6.4 & 18.6 \\
\hline Bachillerato & 1.7 & 28.9 & 16.1 & 9.9 & 6.6 & 6.6 & 5.8 & 5.0 & 10.7 & 8.7 \\
\hline Superior & 4.5 & 26.1 & 16.4 & 9.7 & 9.3 & 4.5 & 6.0 & 5.2 & 12.7 & 5.6 \\
\hline Todos & 3.9 & 28.1 & 15.2 & $I 1.6$ & 6.9 & 5.5 & 4.3 & 3.1 & 8.1 & 13.2 \\
\hline
\end{tabular}

¿Podría miencionar el principal fracaso del gobiemo en estos tres años?

\subsection{Los problemas nacionales y el trabajo del gobierno}

Todos estos datos, tanto la impresión sobre la ausencia de cambios positivos de trascendencia como la creencia que sí han habido bastantes cambios negativos importantes con la gestión de Calderón Sol, pueden servir como indicadores de la magnitud de los problemas que debe enfrentar la administración gubernamental. Para aproximarse mejor a este tema, los encuestadores del IUDOP pidieron a la gente definir con una pregunta abierta cuáles son las dificultades nacionales más importantes. La Figura 1 muestra los resultados a esa pregunta. La res- puesta más sobresaliente fue la delincuencia, que representa el 38.7 por ciento de los problemas mencionados.. De manera muy semejante a las respuestas que salieron como los principales fracasos del actual gobierno, la gran mayoría de los demás temas mencionados por los entrevistados se acerca a los problemas económicos que enfrenta actualmente la ciudadanía salvadoreña. El 19.8 por ciento dijo que la economía misma es el principal problema del país, mientras que otros mencionaron asuntos específicos en el ámbito económico: el desempleo (el 15.3 por ciento), la inflación (8.5 por ciento) y la pobreza (7.9 por ciento). De tal manera que al combinar todas las respuestas relacionadas con la 
economía, en realidad, la mayoría de los encuestados (51.5 por ciento) está preocupada, sobre todo, por los problemas económicos que enfrenta en la actualidad.

Figura 1

Opinión sobre el principal problema del país

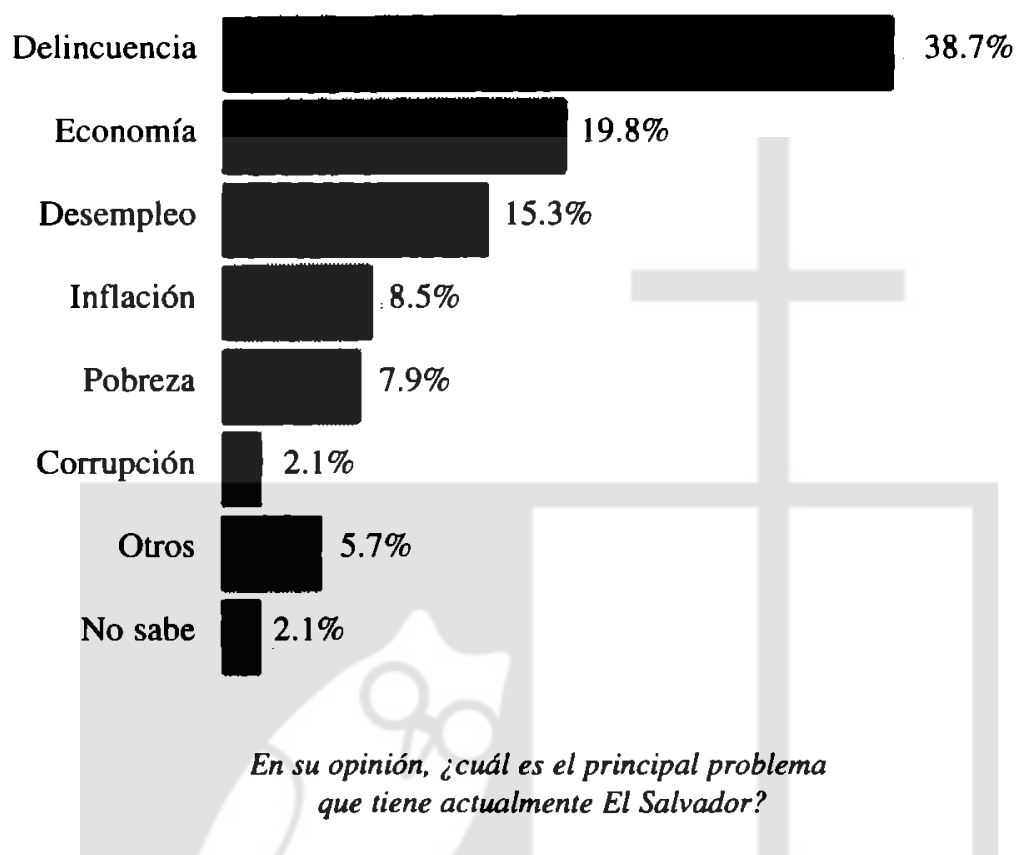

Además de pedir a los ciudadanos que definieran, en sus propias palabras, el principal problema que tiene en la actualidad El Salvador, también se les preguntó si el gobiemo está resolviendo algunas de esas dificultades, específicamente la corupción, la delincuencia y la economía. Como se muestra en el Cuadro 6, la gran mayoría de los ciudadanos cree que el gobierno no está resolviendo los problemas más graves e importantes del país. Tres de cada cuatro participantes en la encuesta dijeron que la gestión gubernamental actual no está resolviendo los problemas ni de la corrupción (74.0 por ciento) ni de la delincuencia (75.3 por ciento); es más, cinco de cada seis (83.6 por ciento) dijeron que el gobierno no está resolviendo el problema económico. Es decir, los problemas que la gente considera como los principales que enfrenta actualmente El Salvador son los mismos que los ciudadanos ven como no resueltos por parte del gobierno de Calderón Sol.
Cuadro 6

Opinión de si el gobierno está resolviendo los problemas del país

(En porcentajes)

\begin{tabular}{lccc}
\hline & \multicolumn{2}{c}{ Opinión } & \\
\cline { 2 - 3 } Problemas & No resuelve & Sí resuelve & \multirow{2}{*}{ No sabe } \\
\hline Corrupción & 74.0 & 20.3 & 5.8 \\
Delincuencia & 75.3 & 21.1 & 3.5 \\
Economía & 83.6 & 13.1 & 3.3 \\
\hline
\end{tabular}

¿Cree Ud. que el gobierno está resolviendo o no los problemas de la compción, la delincuencia y economia?

En cuanto a la economía, el Instituto Universitario de Opinión Pública de la UCA buscó 
indicadores más directos sobre cómo la ciudadanía percibe la situación, tanto del país como de la familia misma desde una perspectiva comparativa. Se preguntó a los ciudadanos entrevistados si creen que la situación económica del país ha mejorado o empeorado con el actual gobierno; los resultados indican que la mayoría (el 51.5 por ciento) sostiene que las condiciones económicas del país han empeorado, en comparación con sólo el 14.5 por ciento que considera que ha mejorado y el 31.5 por ciento que afirma que se mantienen igual. En cambio, la respuesta más común en lo que se refiere a la situación económica familiar es que se ha mantenido sin cambios, respuesta brindada por el 49.0 por ciento de los encuestados. Sin embargo, el 41.4 por ciento cree que su condición doméstica financiera ha empeorado y sólo el 8.5 por ciento percibió que sus condiciones mejoraron. En síntesis, estos datos muestran que muy poca gente considera que el gobierno actual ha mejorado la situación económica nacional y doméstica de los ciudadanos. Sin entrar a valorar la validez de esta percepción casi generalizada, lo cierto es que uno de los principales retos de cualquier gobierno es el desarrollo de las condiciones de vida de sus ciudadanos, esto acontece con el mejoramiento de la situación económica de los mismos como del país; si esto no se cumple, al menos desde la perspectiva de la población, no es extraño que la administración gubernamental tenga muchas dificultades para conseguir una buena evaluación por parte de sus gobernados.

\section{Figura 2}

Opinión sobre la situación económica con el actual gobiemo

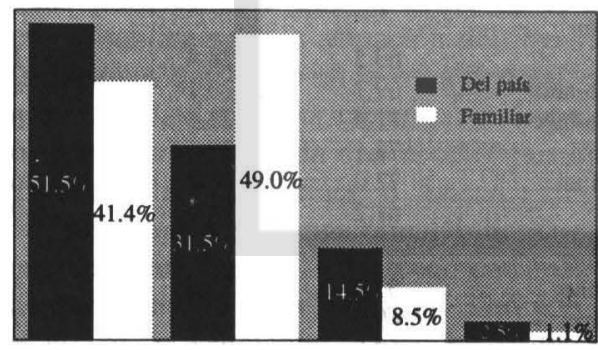

¿Cree Ud. que la situación económica del país ha mejorado o empeorado con el actual gobierno?

¿Su situación económica familiar ha mejorado o empeorado con la gestión del actual gobierno?
Lo anterior ayuda a explicar también otro dato revelado por el sondeo de los tres años de gestión del gobierno de Calderón Sol: todos los asuntos que, de alguna manera, están relacionados con la política económica del gobierno obtienen muy poco apoyo político por parte de los ciudadanía. A pesar de la campaña publicitaria que se ha desplegado a través del último año y las discusiones que se han llevado a cabo en la asamblea legislativa, la gente parece no estar a favor de la privatización de las empresas públicas (ver Figura 3). Sólo la cuarta parte de la ciudadanía (el 23.7 por ciento) está de acuerdo con el proceso de privatización de las compañías estatales como ANTEL y CEL, mientras que más de la mitad de los consultados, el 57.1 por ciento, se manifestó en desacuerdo con tal política.

Figura 3

Opinión sobre el proceso de privatización de las empresas públicas

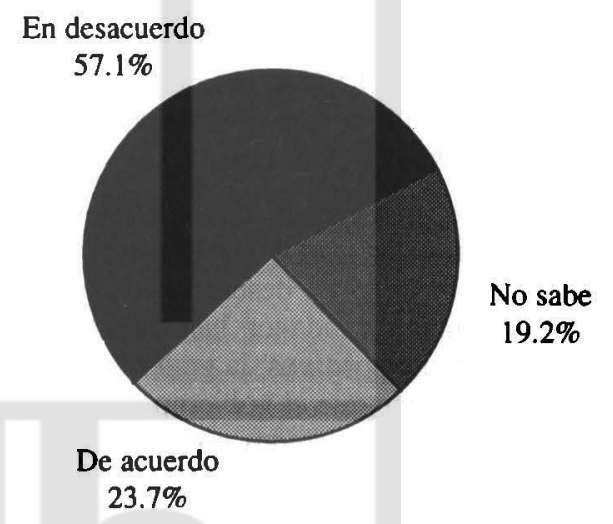

¿Está Ud. de acuerdo o en desacuerdo con el proceso de privatización de las empresas públicas como ANTEL o CEL que está llevando a cabo el gobierno?

Con todo, a pesar del rechazo mayoritario al proceso de privatización que impulsa el gobiemo, es interesante ver que algunos grupos sociales están menos dispuestos que otros a rechazar tal proceso . De hecho, la mayoría de personas que constituye el estrato alto se manifiesta de acuerdo con tal proceso ( el 51.9 por ciento), y sólo el 37.0 por ciento de estas personas se muestra en contra. Es probable que esta diferencia clasista en una opinión tan singular resulte del hecho de que las personas con más recur- 
sos perciben más beneficios a su condición si este proceso se concretiza, mientras que los demás temen pagar precios cada vez más altos o las oportunidades de empleo.

Este asunto está muy relacionado con la manera en que los encuestados perciben al grupo de los más beneficiados por el gobierno actual de Calderón Sol. Al preguntarles si el actual gobierno se preocupa por todos o si, en cambio, sólo se preocupa por ciertos grupos, sólo el 15.3 por ciento de los encuestados respondió que el Ejecutivo sí se preocupa por todos. Aunque algunos mencionaron ciertos grupos específicos como los más beneficiados, por ejemplo, el 6.5 por ciento señaló a los

Todos estos datos revelan que la calificación que los ciudadanos dan al gobierno durante su período corrido de gestión depende de muchos factores sociodemográficos; pero en todo caso, los resultados revelan que aun las calificaciones de los más privilegiados de la sociedad nunca alcanzan un nivel significativamente alto. empresarios y el 4.1 por ciento apuntó a los miembros del partido de ARENA, la gran mayoría de las respuestas coincidió en que el gobierno se interesa sólo por los ricos (el 54.1 por ciento).

En resumen, todos estos datos revelan que los salvadoreños no están muy contentos con la manera en que el gobierno se ha desempeñado en su gestión. Muchos más ciudadanos han notado cambios negativos antes que positivos; la gran mayoría de los salvadoreños no cree que el gobierno esté resolviendo los problemas considerados como los principales en el país; muy pocos ven la situación económica en proceso de recuperación, mientras que muchos la consideran en deterioro; además, la idea de que el gobierno actual sólo se preocupa por algunos grupos de la sociedad y no por todos los salvadoreños aparece muy fuerte y de manera consistente. Los resultados del sondeo de opinión pública del IUDOP muestran, de manera clara, que, hasta la fecha, los salvadoreños no están contentos con el trabajo del actual gobierno de ARENA.

\subsection{El cumplimiento de las promesas}

Interrogados sobre qué tanto el gobierno actual ha cumplido sus promesas de campaña, en sus tres primeros años de trabajo, sólo uno de cada cuatro encuestados dijo que sí está cumpliéndolas (ver el Cuadro 7). En cambio, dos de cada tres salvadorenos piensan que no las está cumpliendo. La insatis- facción por el cumplimiento es más grande aún si se consideran las preferencias partidarias de los encuestados. Los que afirmaron haber votado por un diputado de ARENA en las últimas elecciones estaban lógicamente más dispuestos a decir que el Presidente está cumpliendo sus promesas, con una mayoría del 55.1 por ciento. En cambio, los partidarios del resto de institutos políticos más los entrevistados que no votaron en la elecciones anteriores, pensaban que Calderón Sol no está cumpliendo con lo que prometió durante su campaña electoral.

Más sobresaliente aún es el hecho de que, al cumplir tres de sus cinco años de gestión, los salvadoreños consideraron que el Presidente ya ha tenido suficiente tiempo para cumplir con lo prometido. El 63.4 por ciento de los encuestados respondió que el Dr. Calderón Sol sí tuvo suficiente tiempo para cumplir sus promesas de campaña, mientras que sólo el 31.1 por ciento opinó que el ex-presidente del partido ARENA necesita más tiempo para cumplirlas.

\section{Cuadro 7}

Opinión de si el actual gobierno está cumpliendo con sus promesas según variables (En porcentajes)

\begin{tabular}{lccc}
\hline & \multicolumn{2}{c}{ Opinión } & \\
\cline { 2 - 3 } Variables & No & Sín & No Sabe \\
\hline Estrato & & & \\
Alto & 60.5 & 28.4 & 11.1 \\
Medio-alto & 69.7 & 26.1 & 4.2 \\
Medio-bajo & 71.9 & 23.1 & 5.0 \\
Obrero & 73.1 & 20.9 & 6.0 \\
Marginal & 72.0 & 21.7 & 6.4 \\
Rural & 61.2 & 28.9 & 9.9 \\
Estudios & & & \\
Ninguno & 57.4 & 29.6 & 13.0 \\
Primaria & 69.6 & 23.1 & 7.3 \\
Plan básico & 62.8 & 32.0 & 5.2 \\
Bachillerato & 74.4 & 20.7 & 5.0 \\
Superior & 67.9 & 25.0 & 7.1 \\
Todos & 67.5 & 25.1 & 7.3 \\
\hline
\end{tabular}

¿Cree usted que el actual gobierno de ARENA está cumpliendo con sus promesas? 


\subsection{La evaluación global del gobierno}

Visto todo lo anterior, es momento de exponer los resultados de la evaluación general de los ciudadanos sobre el trabajo del Poder Ejecutivo. Como es la costumbre del IUDOP, ésta se hizo de dos formas. En primer lugar, se preguntó por la actuación de Armando Calderón Sol como Presidente de la República, pidiendo a los encuestados calificar su desempeño como "muy bueno", "bueno", "malo" o "muy malo". En segundo lugar, se pidió a los entrevistados que asignaran un número al desempeño del gobierno dentro de una escala de 0 a 10. Los resultados de la primera evaluación se encuentran en el Cuadro 8.

De acuerdo con este cuadro, la mayoría de las respuestas se centra en las evaluaciones menos extremas en cualquier dirección: el 29.3 por ciento contestó con una evaluación "mala", el 30.6 por ciento contestó que "regular", y el 28.3 por ciento respondió con una evaluación "buena". Sin embargo, la evaluación global es algo más negativa que positiva: las respuestas negativas suman 35.3 por ciento, mientras que las positivas reúnen el 32.2 por ciento. Más aún, aunque la población parece, según estos datos, estar más o menos igualmente dividida en las calificaciones del Presidente, surgen diferencias importantes cuando se consideran los distintos subgrupos de la población encuestada. Considerando, por ejemplo, las distintas zonas del país, se nota que la gente de la zona paracentral califica peor la actuación del Presidente que las personas que viven en la zona oriental; en la zona paracentral, el 47.2 por ciento ofreció una evaluación negativa comparada con el 22.7 por ciento que la calificó de "muy buena" o "buena"; en cambio, los ciudadanos de la zona oriental dieron calificaciones de "muy buena" o "buena" con más frecuencia que "muy mala" o "mala" (el 37.6 y el 29.2 por ciento, respectivamente). También se observan diferencias fuertes en las calificaciones según los estratos socioeconómicos y según la preferencia política partidaria.

Los resultados de la segunda evaluación, pre- sentados en el Cuadro 9, demuestran de nuevo una calificación más negativa que positiva sobre el trabajo de la administración gubernamental. En la escala de 0 o 10, el Ejecutivo recibió una nota promedio de 4.97. Las clases más privilegiadas le dieron una evaluación algo más alta, alcanzando 5.29 por el estrato alto y 5.26 por el estrato medio-alto; en cambio, la nota más baja fue dada por el estrato urbano-marginal, que le asignó sólo 4.59. Se pueden observar también diferencias significativas de las notas según el nivel de estudios (que varían entre 4.58 para los que no tienen educación formal y 5.34 para los que poseen educación superior) y las zonas del país (que varían entre 4.59 en la zona paracentral y 5.37 en la zona oriental). Pero lo más sobresaliente de los datos de la escala de evaluación al gobierno es la diferencia según la edad de los encuestados: hay una clara disparidad entre los jóvenes (nota promedio de 5.22 en las edades 18 a 25 años de edad) y los ancianos (4.67 de los 56 años en adelante); a medida que aumenta la edad, la calificación se vuelve más negativa. Todos estos datos revelan que la calificación que los ciudadanos dan al gobierno durante su período corrido de gestión depende de muchos factores sociodemográficos; pero, en todo caso, los resultados revelan que aun las calificaciones de los más privilegiados de la sociedad nunca alcanzan un nivel significativamente alto.

\section{El principal reto del gobierno}

Cualquier oportunidad para evaluar a la clase dirigente no estaría completa si al mismo tiempo no existe la oportunidad para que los ciudadanos puedan proponer los retos que el gobierno debe, a su juicio, de cumplir. Lógicamente, la mayoría de las sugerencias de la ciudadanía en el sondeo de evaluación del IUDOP se concentró en los mismos asuntos que se señalaron como problemáticos dentro de la gestión del gobiemo. Esto es, la delincuencia y los aspectos relacionados con el desarrollo económico del país. 


\section{Cuadro 8}

Evaluación de la actuación de Calderón durante sus tres años de gobierno según variables (En porcentajes)

\begin{tabular}{|c|c|c|c|c|c|c|}
\hline \multirow[b]{2}{*}{ Variables } & \multicolumn{5}{|c|}{ Evaluación } & \multirow[b]{2}{*}{ No sabe } \\
\hline & Muy mala & Mala & Regular & Buena & Muy Buena & \\
\hline \multicolumn{7}{|l|}{ Zona del país } \\
\hline Occidental & 7.8 & 25.0 & 34.4 & 25.4 & 2.0 & 5.5 \\
\hline Central & 8.6 & 29.3 & 25.7 & 29.3 & 2.1 & 5.0 \\
\hline Paracentral & 4.9 & 42.3 & 25.2 & 20.9 & 1.8 & 4.9 \\
\hline Oriental & 5.2 & 24.0 & 28.4 & 33.9 & 3.7 & 4.8 \\
\hline Metropolitana & 4.9 & 30.5 & 33.6 & 28.9 & 0.5 & 1.6 \\
\hline \multicolumn{7}{|l|}{ Estrato } \\
\hline Alto & 9.9 & 24.7 & 37.0 & 23.5 & 2.5 & 2.5 \\
\hline Medio-alto & 6.1 & 29.1 & 27.3 & 32.1 & 4.2 & 1.2 \\
\hline Medio-bajo & 6.3 & 27.5 & 36.3 & 27.5 & 1.3 & 1.3 \\
\hline Obrero & 5.2 & 30.9 & 28.5 & 30.9 & 0.4 & 4.0 \\
\hline Marginal & 3.8 & 35.0 & 29.3 & 26.1 & 1.3 & 4.5 \\
\hline Rural & 6.4 & 27.9 & 30.1 & 27.2 & 2.2 & 6.2 \\
\hline \multicolumn{7}{|l|}{ Sexo } \\
\hline Masculino & 6.5 & 30.2 & 30.3 & 28.3 & 1.5 & 3.2 \\
\hline Femenino & 5.6 & 28.6 & 30.8 & 28.3 & 2.2 & 4.6 \\
\hline \multicolumn{7}{|l|}{ Edad } \\
\hline 18 a 25 años & 4.3 & 25.7 & 28.2 & 35.0 & 3.4 & 3.4 \\
\hline 26 a 40 años & 4.9 & 27.8 & 33.8 & 28.1 & 1.5 & 4.0 \\
\hline 41 a 55 años & 9.4 & 35.2 & 29.1 & 23.0 & $0.4 r$ & 2.9 \\
\hline 56 años y más & 7.4 & 31.8 & 28.4 & 23.9 & 2.3 & 6.3 \\
\hline \multicolumn{7}{|l|}{ Estudios } \\
\hline Ninguno & 8.6 & 35.8 & 24.1 & 23.5 & 0.0 & 8.0 \\
\hline Primaria & 5.4 & 32.1 & 30.7 & 25.0 & 1.1 & 5.7 \\
\hline Plan básico & 7.0 & 19.2 & 27.8 & 28.5 & 4.1 & 3.5 \\
\hline Bachillerato & 5.8 & 26.0 & 32.2 & 31.8 & 2.5 & 1.7 \\
\hline Superior & 4.9 & 31.0 & 28.4 & 32.1 & 2.2 & 1.5 \\
\hline \multicolumn{7}{|l|}{ Voto por diputado } \\
\hline Ninguno & 0.0 & 50.0 & 30.0 & 20.0 & 0.0 & 0.0 \\
\hline ARENA & 1.3 & 12.7 & 30.4 & 48.7 & 4.4 & 2.5 \\
\hline FMLN & 7.7 & 38.3 & 27.6 & 24.0 & 1.0 & 1.5 \\
\hline PDC & 4.0 & 40.0 & 28.0 & 20.0 & 4.0 & 4.0 \\
\hline Otros & 9.8 & 40.2 & 23.2 & 24.4 & 0.0 & 0.0 \\
\hline No sabe/no responde & 3.5 & 24.4 & 41.3 & 23.9 & 2.5 & 4.5 \\
\hline Todos & 6.0 & 29.3 & 30.6 & 28.3 & 1.9 & 3.9 \\
\hline
\end{tabular}

¿Cómo evalúa la actuación del presidente Calderón a lo largo de sus tres años de gobierno, muy buena, buena, mala o muy mala? 


\section{Cuadro 9}

Calificación del gobierno según variables

\begin{tabular}{|c|c|c|}
\hline Variables & $\mathrm{N}$ & Promedio \\
\hline \multicolumn{3}{|l|}{ Estrato } \\
\hline Alto & 79 & 5.29 \\
\hline Medio-alto & 161 & 5.26 \\
\hline Medio-bajo & 158 & 5.02 \\
\hline Obrero & 242 & 4.92 \\
\hline Marginal & 148 & 4.59 \\
\hline Rural & 375 & 4.93 \\
\hline \multicolumn{3}{|l|}{ Estudios } \\
\hline Ninguno & 136 & 4.58 \\
\hline Primaria & 347 & 4.64 \\
\hline Plan básico & 169 & 5.18 \\
\hline Bachillerato & 240 & 5.07 \\
\hline Superior & 266 & 5.34 \\
\hline \multicolumn{3}{|l|}{ Sexo } \\
\hline Masculino & 565 & 5.01 \\
\hline Femenino & 598 & 4.92 \\
\hline \multicolumn{3}{|l|}{ Edad } \\
\hline 18 a 25 años & 315 & 5.22 \\
\hline 26 a 40 años & 460 & 5.00 \\
\hline 41 a 56 años & 232 & 4.75 \\
\hline 56 años y más & 156 & 4.67 \\
\hline \multicolumn{3}{|l|}{ Zona del pais } \\
\hline Occidental & 246 & 4.93 \\
\hline Central & 129 & 4.70 \\
\hline Paracentral & 157 & 4.59 \\
\hline Oriental & 254 & 5.37 \\
\hline Metropolitana & 377 & 4.96 \\
\hline Todos & 1163 & 4.97 \\
\hline
\end{tabular}

En una escala de 0 a 10, ¿qué nota le daría al gobierno de Armando Calderón Sol por su desempeño hasta ahora?

El Cuadro 10 presenta un resumen de las respuestas acerca de la principal tarea que el gobierno actual debe enfrentar en el próximo año de su gestión. Según estos resultados, uno de cada cinco encuestados (el 20 por ciento) cree que el combate de la criminalidad debe ser la principal tarea del gobierno. Como fue el caso en las preguntas sobre el principal problema del país y el principal fracaso del gobierno actual de ARENA, casi todas las demás respuestas tienen que ver con la situación económica en que los salvadoreños se encuentran. Sumando las respuestas de crear fuentes de trabajo (el 26.5 por ciento), mejorar la economía (el 12.2 por ciento), combatir la pobreza (el 9.3 por ciento) y controlar los precios (el 8.3 por ciento), se logra un total del 56.3 por ciento de los encuestados; una mayoría de la población que cree que la tarea más importante del gobiemo actual tiene relación con el bienestar económico de la ciudadanía de una manera u otra. 


\section{Cuadro 10}

Opinión sobre la principal tarea del gobierno en su próximo año de gestión según variables (En porcentajes)

\begin{tabular}{|c|c|c|c|c|c|c|c|c|c|c|}
\hline \multirow[b]{2}{*}{ Variables } & \multicolumn{8}{|c|}{ Principal tarea } & \multirow[b]{2}{*}{$\begin{array}{l}\text { Otras } \\
\text { tareas }\end{array}$} & \multirow[b]{2}{*}{ No sabe } \\
\hline & $\begin{array}{l}\text { Crear } \\
\text { empleo }\end{array}$ & $\begin{array}{c}\text { Combatir } \\
\text { crimen }\end{array}$ & $\begin{array}{l}\text { Mejorar } \\
\text { economía }\end{array}$ & $\begin{array}{l}\text { Combatir } \\
\text { pobreza }\end{array}$ & $\begin{array}{l}\text { Controlar } \\
\text { precios }\end{array}$ & $\begin{array}{l}\text { Combatir } \\
\text { comupción }\end{array}$ & $\begin{array}{l}\text { Cumplir } \\
\text { promesas }\end{array}$ & $\begin{array}{l}\text { Problemas } \\
\text { politicos }\end{array}$ & & \\
\hline \multicolumn{11}{|l|}{ Estrato } \\
\hline Alto & 19.8 & 28.4 & 16.0 & 8.6 & 2.5 & 1.2 & 1.2 & 3.7 & 16.0 & 2.5 \\
\hline Medio-alto & 18.8 & 24.8 & 20.0 & 6.7 & 4.8 & 4.2 & 1.2 & 6.1 & 12.1 & 1.2 \\
\hline Medio-bajo & 28.1 & 18.8 & 13.1 & 5.6 & 6.9 & 6.3 & 3.1 & 2.5 & 13.8 & 1.9 \\
\hline Obrero & 29.3 & 21.7 & 13.7 & 12.0 & 5.6 & 4.8 & 2.8 & 1.2 & 4.4 & 4.4 \\
\hline Marginal & 30.6 & 12.1 & 7.6 & 14.6 & 8.3 & 1.9 & 1.9 & 1.3 & 8.9 & 12.7 \\
\hline Rural & 27.2 & 18.8 & 8.9 & 8.1 & 13.1 & 1.2 & 2.0 & 0.7 & 7.2 & 12.8 \\
\hline \multicolumn{11}{|l|}{ Estudios } \\
\hline Ninguno & 28.4 & 12.3 & 6.2 & 13.0 & 14.2 & 2.5 & 1.2 & 1.9 & 5.6 & 14.8 \\
\hline Primaria & 27.7 & 15.8 & 8.4 & 10.1 & 10.9 & 2.7 & 3.0 & 1.4 & 8.4 & 11.7 \\
\hline Plan básico & 34.3 & 23.8 & 8.7 & 10.5 & 7.0 & 0.6 & 1.2 & 0.6 & 5.8 & 7.6 \\
\hline Bachillerato & 23.6 & 24.8 & 17.4 & 7.9 & 5.0 & 4.1 & 2.9 & 0.8 & 10.3 & 3.3 \\
\hline Superior & 21.6 & 23.9 & 19.0 & 6.7 & 4.1 & 4.9 & 1.5 & 5.2 & 12.3 & 0.7 \\
\hline \multicolumn{11}{|l|}{ Sexo } \\
\hline Masculino & 27.9 & 18.9 & 13.6 & 9.2 & 5.6 & 3.6 & 2.0 & 1.9 & 10.9 & 6.3 \\
\hline Femenino & 25.2 & 21.0 & 11.0 & 9.4 & 10.8 & 2.7 & 2.2 & 2.2 & 7.1 & 8.4 \\
\hline \multicolumn{11}{|l|}{ Edad } \\
\hline 18 a 25 años & 23.5 & 27.9 & 9.6 & 9.0 & 7.7 & 3.7 & 2.5 & 1.2 & 7.1 & 7.7 \\
\hline 26 a 40 años & 27.4 & 19.8 & 13.9 & 9.3 & 7.2 & 2.7 & 2.3 & 2.3 & 7.2 & 7.8 \\
\hline 41 a 55 años & 29.1 & 16.0 & 13.9 & 6.6 & 8.6 & 3.3 & 1.6 & 2.5 & 12.3 & 6.1 \\
\hline 56 años y más & 26.1 & 11.4 & 10.2 & 13.6 & 11.9 & 2.8 & 1.7 & 2.3 & 12.5 & 7.4 \\
\hline \multicolumn{11}{|c|}{ Zona del país } \\
\hline Occidental & 27.3 & 18.4 & 14.1 & 7.8 & 7.0 & 0.8 & 3.1 & 1.2 & 11.7 & 8.6 \\
\hline Central & 28.6 & 22.1 & 12.1 & 8.6 & 10.7 & 1.4 & 1.4 & 0.0 & 7.1 & 7.9 \\
\hline Paracentral & 30.7 & 22.7 & 10.4 & 9.8 & 9.2 & 2.5 & 1.8 & 2.5 & 6.1 & 4.3 \\
\hline Oriental & 27.7 & 18.1 & 5.5 & 8.1 & 11.1 & 3.3 & 1.5 & 3.7 & 9.6 & 11.4 \\
\hline Metropolitana & 22.7 & 20.4 & 16.5 & 11.1 & 5.9 & 5.4 & 2.3 & 2.1 & 8.5 & 4.9 \\
\hline Todos & 26.5 & 20.0 & 12.2 & 9.3 & 8.3 & 3.1 & 2.1 & 2.1 & 9.0 & 7.4 \\
\hline
\end{tabular}

En su opinión, ¿cuál es la principal tarea que debe enfrentar el gobierno en el próximo año de gestión?

\section{El rumbo de El Salvador}

En este sondeo, el IUDOP preguntó a los entrevistados sobre "el rumbo" general que tiene el país actualmente. Como puede verse en el Cuadro 11, tres de cada cuatro encuestados (el 76.5 por ciento) opinaron que El Salvador necesita un cambio de rumbo, mientras que sólo la quinta parte (el 21.3 por ciento) sostuvieron que el país va por buen camino. Hay diferencias muy fuertes según los sectores sociales de estrato y educación en estas opiniones: en los estratos, los resultados se mueven en un rango que va desde el 67.9 por ciento en la clase alta hasta el $\mathbf{8 7 . 9}$ por ciento en la clase marginal urbana, mientras que en los niveles de estudio también hubo diferencias en las opiniones. Sin embargo, lo más sobresaliente de estos resultados es que la valoración sobre la necesidad 
de un cambio en el rumbo del país, es una opinión mantenida por la gran mayoría de ciudadanos, lo que estaría mostrando los niveles de insatisfacción ciudadana con las condiciones actuales del país.

\section{Cuadro 11}

Opinión sobre si El Salvador va por buen camino o si necesita cambio, según variables (En porcentajes)

\begin{tabular}{ccc}
\hline & \multicolumn{2}{c}{ Opinión } \\
\cline { 2 - 3 } Variables & $\begin{array}{c}\text { Buen } \\
\text { camino }\end{array}$ & $\begin{array}{c}\text { Necesita } \\
\text { cambio }\end{array}$
\end{tabular}

$\begin{array}{lrrr}\text { Estrato } & & & \\ \text { Alto } & 30.9 & 67.9 & 1.2 \\ \text { Medio-alto } & 20.0 & 76.4 & 3.6 \\ \text { Medio-bajo } & 17.5 & 79.4 & 3.1 \\ \text { Obrero } & 10.8 & 84.7 & 4.4 \\ \text { Marginal } & 8.3 & 87.9 & 3.8 \\ \text { Rural } & 17.3 & 75.1 & 7.7 \\ & & & \\ \text { Estudios } & & & \\ \text { Ninguno } & 16.7 & 75.3 & 8.0 \\ \text { Primaria } & 12.5 & 80.2 & 7.3 \\ \text { Plan básico } & 17.4 & 79.7 & 2.9 \\ \text { Bachillerato } & 14.0 & 82.6 & 3.3 \\ \text { Superior } & 21.3 & 76.5 & 2.2 \\ & & & \\ \text { Todos } & 21.3 & 76.5 & 2.2 \\ \end{array}$

En general, ¿usted piensa que El Salvador va por buen camino o que necesita un cambio?

\section{La evaluación del gobierno en perspectiva}

Todo lo anterior muestra una ciudadanía muy insatisfecha con el gobierno actual del presidente Calderón Sol. Es interesante notar, sin embargo, que estos sentimientos no sólo parecen ser coyunturales o pasajeros. El Cuadro 12 presenta las opiniones y evaluaciones del gobierno desde una perspectiva comparativa, tanto a los cien días, a un año, dos años, y ahora a tres años de gestión. Como se ve muy claramente por la serie de datos, los salvadoreños se han mostrado insatisfechos con la actual administración desde hace tres años. En la mayoría de las preguntas, sus opiniones han permanecido casi igual durante toda la gestión del Ejecutivo. Los problemas de la criminalidad y la economía han sido y siguen siendo las principales preocupaciones de la población; problemas que, en buena medida, son atribuidos a la política misma o al desempeño del gobierno. Es interesante ver cómo después de la evaluación de los primeros cien días de gobierno, la opinión sobre la falta de cumplimiento de las promesas se elevó a dos tercios de la población, porcentaje que no ha variado a través de los años. Ello refuerza precisamente la tesis de que la evaluación que los salvadoreños mantienen sobre su gobierno se ha asentado y no ha variado de manera significativa desde prácticamente el primer año de gobierno. Lo que sí parece haber aumentado lentamente es la impresión de que el Ejecutivo no ha podido resolver los problemas principales, esto junto con la opinión de que el presidente ya ha tenido tiempo suficiente para cumplir con sus promesas.

\section{A manera de conclusión}

El cuarto sondeo para evaluar el trabajo del gobierno, en este caso al cumplirse los tres primeros años de gestión, revela que aquél continúa siendo criticado por la mayoría de la población salvadoreña. La mayor parte de los resultados de esta encuesta indica que los salvadoreños siguen insatisfechos con la gestión del Presidente y de su gabinete ministerial, más que nada en cuanto a economía y delincuencia.

Sin embargo, y como ha venido ocurriendo desde la evaluación del segundo año de gobierno, el sondeo presenta una opinión más compleja de la ciudadanía. Las críticas hacia la Presidencia vienen de todos los sectores sociales, sólo que en distintos puntos. Mientras unos, los más pobres, acusan al gobierno de sólo preocuparse por los ricos y por implementar las políticas económicas en los últimos tres años; otros, la clase media, señalan la falta de cambios o la incapacidad para cumplir las promesas de campaña. Incluso, los sectores de mejor situación socioeconómica señalan aspectos como la delincuencia y la corrupción como talones de aquiles de la administración gubernamental.

De manera general, la evaluación también tiene sus matices. Por un lado, los ciudadanos salvadoreños mantienen las opiniones sobre los principales problemas del país y critican directamente al Ejecutivo por los mismos; pero por otro lado, no ven tantos cambios negativos en la realidad como los veían el año pasado y su evaluación general del gobierno no ha descendido tan significativamente. La evaluación sigue siendo negativa, de 


\section{Cuadro 12}

Resultados comparativos de las evaluaciones del gobierno a cien días, un año, dos años y tres años

\begin{tabular}{lcccc}
\hline Opinión & 100 días & 1 año & 2 años & 3 años \\
\hline La delincuencia como problema & 40.8 & 35.0 & 36.1 & 38.7 \\
Lo económico como problema & 49.9 & 55.2 & 53.5 & 51.5 \\
Evaluación mala o muy mala del presidente & 23.9 & 41.3 & 36.1 & 35.3 \\
Sí ha notado cambios positivos & 31.0 & 34.1 & 46.0 & 42.1 \\
Sí ha notado cambios negativos & 52.0 & 77.3 & 71.2 & 65.2 \\
Gobierno no cumple con sus promesas & 56.7 & 67.5 & 66.3 & 67.5 \\
El gobierno ha tenido tiempo para cumplir & 21.0 & 43.6 & 52.2 & 63.4 \\
Gobierno no resuelve la corrupción & 43.1 & 61.6 & 63.9 & 74.0 \\
Gobierno no resuelve la economía & - & - & 81.0 & 83.6 \\
Gobierno no resuelve la delincuencia & - & - & 66.5 & 75.3 \\
La situación económica ha empeorado & 48.0 & 56.7 & 61.6 & 51.5 \\
El gobierno sólo se preocupa por los ricos & 42.2 & 47.9 & 42.8 & 54.1 \\
Opinión de que El Salvador necesita un cambio & - & 77.3 & 77.4 & 79.0 \\
Lo económico como el mayor reto del gobierno en el futuro & - & 58.4 & 53.9 & 58.1 \\
& & & & \\
Nota de evaluación al gobierno & 6.04 & 4.96 & 5.12 & 4.97 \\
\hline
\end{tabular}

Fuente: IUDOP, 1994, 1995, 1996, 1997.

eso no parece haber duda, pero, la intensidad de la misma no está creciendo como para pensar en un

gobierno que deberá enfrentar peores condiciones en sus dos años que le restan de trabajo. 